\title{
Predictors of Left Ventricular Diastolic Dysfunction in Metabolic Syndrome: Gender Differences
}

\author{
Ewa Kruszyńska ${ }^{1}$, Michaela Kozakova ${ }^{2}$, Maria Łoboz Rudnicka ${ }^{1}$, Carmela Morizzo ${ }^{3}$, Carlo Palombo ${ }^{3}$, Krystyna Łoboz Grudzień ${ }^{4}$ and Joanna Jaroch $^{4}$ \\ ${ }^{1}$ Department of Cardiology, T. Marciniak Hospital, Emergency Medicine Center, Wroclaw, Poland \\ ${ }^{2}$ Department of Clinical and Experimental Medicine, University of Pisa, Italy \\ ${ }^{3}$ Department of Surgical, Medical and Molecular Pathology and Critical Care Medicine, University of Pisa, Italy \\ ${ }^{4}$ Department of Clinical Science, Faculty of Health Sciences, Wroclaw Medical University, Poland
}

*Corresponding author: Joanna Jaroch, Department of Clinical Science, Faculty of Health Sciences, Wroclaw Medical University, Poland, Tel: +48713064702; E-mail: j.jaroch@wp.pl

Received date: Nov 26, 2018; Accepted date: Dec 03, 2018; Published date: Dec 11, 2018

Copyright: ( $) 2018$ Kruszyńska E, et al. This is an open-access article distributed under the terms of the Creative Commons Attribution License, which permits unrestricted use, distribution, and reproduction in any medium, provided the original author and source are credited.

\begin{abstract}
Background: Little is known about the sex-specific differences in predictors of left ventricular diastolic dysfunction in metabolic syndrome. One of the novel paradigms include the role of arterial stiffness in the pathogenesis of left ventricular diastolic dysfunction. There are some differences in arterial stiffness in patients with metabolic syndrome. We hypothesized that arterial stiffness may play a different role in the etiology of diastolic dysfunction in men and women with metabolic syndrome, acting independently from other established predictors of this condition besides the components of metabolic syndromes, such as age, left ventricular hypertrophy and systolic function. The aim of the study was to analyze the sex-specific differences in arterial stiffness as predictors of diastolic dysfunction in metabolic syndrome. Methods: The study included 131 patients from 2 centers in Poland and Italy (60 women and 71 men, mean age $53.7 \pm 6.7$ years) with metabolic syndrome and no history of cardiovascular disease. All patients underwent detailed echocardiography and high-resolution echo-tracking of carotid arteries with the evaluation of arterial stiffness. Results: In the multivariate analysis, relative wall thickness was independently associated with diastolic dysfunction occurrence in women with metabolic syndrome $(P=0.012)$, while independent predictors of diastolic dysfunction in men were relative wall thickness $(P=0.08)$, left ventricular mass index $(P=0.001)$ and arterial compliance $(P=0.004)$. Conclusions: There are sex-differences in left ventricular diastolic dysfunction predictors in metabolic syndrome. Besides left ventricular hypertrophy as a powerful determinant of left ventricular diastolic dysfunction in both men and women with metabolic syndrome, also arterial compliance as a measure of arterial stiffness was independently associated with left ventricular diastolic dysfunction occurrence in men but not in women with metabolic syndrome.
\end{abstract}

Keywords: Ventricular diastolic; Metabolic syndrome; Pathophysiologic mechanism; Left ventricular hypertrophy

\section{Introduction}

Metabolic Syndrome (MS) is an independent predictor of cardiovascular diseases [1]. The cardiovascular risk linked to MS is partly explained by increased prevalence and extent of Target Organ Damage (TOD), such as preclinical carotid atherosclerosis and Left Ventricular (LV) remodeling including Left Ventricular Hypertrophy (LVH) and Diastolic Dysfunction (DD) [2,3], which altogether are considered precursors of clinical cardiovascular events.

Adverse prognostic impact of MS might be greater in women comparing to men [4,5]. Still little is known about sex-specific differences in the predictors of diastolic dysfunction of the Left Ventricle (LV DD) in Metabolic Syndrome. One of the novel paradigms of heart failure with preserved $\mathrm{EF}(\mathrm{HfpEF})$ includes the role of Arterial Stiffness (AS) in the pathogenesis of LV DD [6-10]. Some differences in AS in men and women with MS have been described [11-13]. The hypothesis of our study is that AS may play a different role in the pathophysiologic mechanism underlying LV DD in men and women with MS and that this relationship is independent of established predictors of LV DD such as age, LVH and LV systolic function, besides the components of MS. The aim of the study was to check for the sex-specific differences in AS as predictors-of LV DD in MS.

\section{Patients and Methods}

A total of 159 patients with metabolic syndrome defined according to NCEP-ATP III were enrolled to the study from Department of Cardiology, T Marciniak Hospital, Wroclaw, Poland and Department of Surgical, Medical and Molecular Pathology and Critical Care Medicine; Division of Anesthesiology, University of Pisa, Italy. Patients were selected from a larger population referred to cardiological evaluation for high blood pressure, dyspnoea or atypical chest pain, resulted free of clinical cardiovascular disease (myocardial ischemia and/or previous myocardial infarction, heart failure, TIA/stroke), and with echocardiography showing ejection fraction $(\mathrm{EF})>55 \%$ and no evidence of regional wall motion abnormalities, cardiomyopathy, pericardial disease, or valve dysfunctions.

After excluding subjects with an inadequate quality of echocardiographic or echo tracking data, the final study population consisted of 131 pts (60 women and 71 men, mean age $53.7 \pm 6.7$ years). All selected patients underwent complete 2D, M-mode, and Doppler echocardiography and carotid ultrasound, including high- 
resolution eTracking. This study was approved by the Ethics Committee of Wroclaw University of Medicine and University of Pisa; written informed consent was sought from each participant.

\section{Echocardiography}

Detailed two-dimensional Doppler and tissue Doppler echocardiographic recordings (Alpha 10; HITACHI-ALOKA, Tokyo, Japan) were performed in each patient. Volumes were assessed with the Teichholz method. LV Stroke Volume (SV) normalized for Pulse Pressure (PP) was used as a measure of total arterial compliance (SV/ PP). End-diastolic wall thickness (Interventricular Septum [IVS] and Posterior Wall $[\mathrm{PW}]$ ) and Left Ventricle cavity diameter (LV EndDiastolic Diameter [EDD]) were used to calculate LV Mass (LVM) from the formula introduced by Devereux et al. [14] and then normalized for BSA to obtain LV Mass Index (LVMI). Relative Wall Thickness (RWT) was assessed from the formula: RWT=2PW/EDD. The criterium for Left Ventricular Hypertrophy (LVH) was LVMI $>110$ $\mathrm{g} / \mathrm{m}^{2}$ for women or- $>125 \mathrm{~g} / \mathrm{m}^{2}$ for men.

\section{Assessment of LV systolic and diastolic function}

LV systolic function was evaluated by Ejection Fraction (EF) calculated with Teichholtz method, Midwall Fractional Shortening (MFS) according to de Simone et al. [15] and tissue Doppler-derived peak longitudinal systolic shortening velocity $\left(S^{\prime}\right)$ from apical fourchamber view at the lateral and septal mitral annulus and then averaged [16]. LV diastolic function was assessed based on conventional Doppler mitral inflow and tissue Doppler of the mitral annulus, according to recommendations [16]. Early (E) and late (A) Velocities, E/A ratio, and E Wave Deceleration Time (DTE) were determined on the basis of transmitral pulse wave Doppler. Tissue Doppler parameters, that is, early $\left(\mathrm{e}^{\prime}\right)$ and late $\left(\mathrm{a}^{\prime}\right)$ Diastolic Mitral Annular Velocities, their ratio $\left(\mathrm{e}^{\prime} / \mathrm{a}^{\prime}\right)$, were measured at septal and lateral sides of the mitral annulus and then averaged. E/e' was considered as an index of LV filling pressure.

\section{Integrated assessment of arterial function}

Vascular ultrasonography of the right common carotid artery was performed with an HITACHI-ALOKA Alpha 10 device with an integrated and automated ultrasonographic, Doppler, and highresolution echo-tracking system. After visualization of the IntimaMedia Complex (IMT) of anterior and posterior arterial walls in longitudinal axis with a maximal internal diameter, an echo-tracking sample was positioned at the blood-intima interface, with $1 \mathrm{kHz}$ sampling rate for detection of carotid diameter changes. Diameter changes follow changes in intravascular pressure, which enables a conversion of carotid diameter waveform changes into arterial pressure waveforms by calibrating peak and minimal values to systolic and diastolic brachial blood pressures [17]. The data for minimum five beats were averaged. The following AS parameters were evaluated online [10]:

1. $\quad \beta$ (beta stiffness index), a ratio of the natural logarithm of systolic/diastolic blood pressure to the relative change in the arterial diameter: $\beta=\ln (\mathrm{Ps} / \mathrm{Pd}) /[(\mathrm{Ds}-\mathrm{Dd}) / \mathrm{Dd}]$, where $\ln$ is the natural logarithm, Ps the systolic blood pressure, Pd the diastolic blood pressure, Ds the arterial systolic diameter, and Dd is the arterial diastolic diameter

2. Epsilon (Ep): Young's modulus, also referred to the pressurestrain elasticity modulus: $\mathrm{Ep}=(\mathrm{Ps}-\mathrm{Pd}) /[(\mathrm{Ds}-\mathrm{Dd}) / \mathrm{Dd}]$
3. Arterial compliance (AC): AC, from the arterial cross-sectional area and blood pressure: $\mathrm{AC}=\pi(\mathrm{Ds} \times \mathrm{Ds}-\mathrm{Dd} \times \mathrm{Dd}) /[4 \times(\mathrm{Ps}-$ Pd)]

4. Pulse wave velocity $(\mathrm{PWV} \beta)$ : one-point $\mathrm{PWV}$, from the time delay between two adjacent distension waveforms, based on the water hammer equation and using the $\beta: P W V \beta:=\div(\beta: \mid P / 2 \backslash r)$

Where P-diastolic blood pressure, r-blood density $\left(1,050 \mathrm{~kg} / \mathrm{m}^{3}\right)$.

The reproducibility of the measurements mentioned earlier has been reported elsewhere [18]. IMT was determined in line with the established standards. In addition, serum concentrations of glucose and lipids were determined in all the study subjects.

\section{Statistical Analysis}

Mean and standard deviations were calculated for quantitative variables and percentages for qualitative variables. As all variables were not normally distributed, differences between groups were tested by Mann-Whitney test for quantitative variables and by a $c^{2}$ test for percentages of qualitative variables. The statistical significance was at $\mathrm{p}<0.05$ (two-sided tests), and for multiple testing, we used a statistical significance of $\mathrm{p}<0.01)$. For quantitative variables showing a statistically significant difference between the two groups, ReceiverOperating Characteristic (ROC) curves were used to calculate the cutoff values optimized to reach the best compromise in the prediction of diastolic dysfunction. Optimal cut-off was defined as a threshold when the sum of sensitivity and specificity was maximum. A multivariable logistic regression analysis was performed considering as dependent variables the occurrence of diastolic dysfunction. All the variables with a significant value $<0.25$ at univariate analysis were included into the model. The stepwise method with backward elimination was used, and Odds Ratios (OR) with 95\% CIs was calculated. The model was evaluated using the Hosmer-Lemeshow test.

\section{Results}

\section{Clinical characteristics}

There were no significant differences in the mean values of age, Body Mass Index (BMI), systolic (SBP) and Mean Blood Pressure (MBP in male and female patients with MS. Women presented with higher mean values of Heart Rate $(\mathrm{HR} ; \mathrm{P}=0.043)$ and $\mathrm{DBP}(\mathrm{P}=0.04)$ and men with higher mean values of waist circumference $(\mathrm{P}=0.001)$. The prevalence of diabetes mellitus was higher in men than in women $(\mathrm{P}<0.001)$ and mean values of glucose were higher in men compared to women $(\mathrm{P}<0.001)$. Women had higher mean values of HDL $(\mathrm{P}<0.001)$ and lower mean values of triglycerides $(\mathrm{P}<0.001)$ (Table1).

\begin{tabular}{|c|c|c|c|c|}
\hline \multirow[b]{3}{*}{ Clinical parameters } & \multirow{3}{*}{$\begin{array}{l}\text { All } \\
N=131\end{array}$} & \multicolumn{2}{|l|}{ Sex } & \multirow[t]{3}{*}{ W vs. $M$} \\
\hline & & Women & Men & \\
\hline & & $\mathrm{N}=60$ & $\mathrm{~N}=71$ & \\
\hline Age (years) $M \pm S D$ & $53.7 \pm 11.6$ & $54.6 \pm 12.7$ & $52.9 \pm 10.5$ & $P=0.216$ \\
\hline $\mathrm{BMI}\left(\mathrm{kg} / \mathrm{m}^{2}\right) \mathrm{M} \pm \mathrm{SD}$ & $31.8 \pm 4.5$ & $32.3 \pm 5.3$ & $31.4 \pm 3.7$ & $p=0.647$ \\
\hline$H R(1 / \min ) M \pm S D$ & $70.3 \pm 10.4$ & $72.0 \pm 10.2$ & $68.9 \pm 10.3$ & $p=0.043$ \\
\hline $\mathrm{SBP}(\mathrm{mm} \mathrm{Hg}) \mathrm{M} \pm \mathrm{SD}$ & $136.2 \pm 14.7$ & $\begin{array}{ll}137.2 & \pm \\
14.9 & \end{array}$ & $135.5 \pm 14.7$ & $p=0.443$ \\
\hline $\mathrm{DBP}(\mathrm{mm} \mathrm{Hg}) \mathrm{M} \pm \mathrm{SD}$ & $78.9 \pm 10.2$ & $80.8 \pm 10.2$ & $77.3 \pm 9.9$ & $p=0.043$ \\
\hline
\end{tabular}


Citation: Kruszyńska E, Kozakova M, Rudnicka ML, Morizzo C, Palombo C, et al. (2018) Predictors of Left Ventricular Diastolic Dysfunction in Metabolic Syndrome: Gender Differences. J Metabolic Synd 7: 244. doi:10.4172/2167-0943.1000244

Page 3 of 8

\begin{tabular}{|c|c|c|c|c|}
\hline $\mathrm{MBP}(\mathrm{mm} \mathrm{Hg}) \mathrm{M} \pm \mathrm{SD}$ & $98.0 \pm 10.8$ & $99.6 \pm 10.7$ & $96.6 \pm 10.7$ & $p=0.079$ \\
\hline $\begin{array}{l}\text { Glucose }(\mathrm{mg} / \mathrm{dL}) \mathrm{M} \pm \\
\text { SD }\end{array}$ & $132.3 \pm 48.9$ & $\begin{array}{l}111.5 \quad \pm \\
36.0\end{array}$ & $149.9 \pm 51.7$ & $p<0.001$ \\
\hline Waist $(\mathrm{cm}) \mathrm{M} \pm \mathrm{SD}$ & $\begin{array}{l}n=122102.8 \\
\pm 12.9\end{array}$ & $\begin{array}{l}n=60 \quad 99.0 \\
\pm 10.7\end{array}$ & $\begin{array}{l}n=62 \quad 106.5 \\
\pm 13.8\end{array}$ & $p=0.001$ \\
\hline $\mathrm{HDL}(\mathrm{mg} / \mathrm{dL}) \mathrm{M} \pm \mathrm{SD}$ & $\begin{array}{l}n=127 \quad 48.0 \\
\pm 13.1\end{array}$ & $\begin{array}{l}n=57 \quad 53.4 \\
\pm 13.9\end{array}$ & $\begin{array}{l}\mathrm{n}=7043.7 \pm \\
10.7\end{array}$ & $p<0.001$ \\
\hline $\mathrm{TG}(\mathrm{mg} / \mathrm{dL}) \mathrm{M} \pm \mathrm{SD}$ & $\begin{array}{l}n=123159.9 \\
\pm 100.0\end{array}$ & $\begin{array}{l}n=54133.1 \\
\pm 86.9\end{array}$ & $\begin{array}{l}n=69 \quad 180.9 \\
\pm 105.1\end{array}$ & $p<0.001$ \\
\hline
\end{tabular}

\section{Echocardiographic characteristics}

Women had significantly lower mean values of LV dimensions and volumes, such as $\operatorname{EDD}(\mathrm{P}<0.001)$, ESD $(\mathrm{P}=0.001)$, EDV $(\mathrm{P}<0.001)$, ESV $(\mathrm{P}<0.001)$, and $\mathrm{SV}(\mathrm{P}<0.001)$ than men (Table 2$)$.Mean values of LVMI were higher in men than in women $\left(108.79\right.$ vs. $\left.83.2 \mathrm{~g} / \mathrm{m}^{2}, \mathrm{P}<0.001\right)$ (Table 2).

Table 1: Clinical characteristics of the study groups.

\begin{tabular}{|c|c|c|c|c|}
\hline \multirow[b]{2}{*}{ Echocardiographic variable } & \multirow{2}{*}{$\begin{array}{l}\text { All } \\
\mathrm{N}=131\end{array}$} & \multicolumn{2}{|l|}{ Sex } & \multirow[t]{2}{*}{ W vs. M } \\
\hline & & Women $\mathbf{N}=60$ & Men $\mathrm{N}=71$ & \\
\hline$P W(m m) M \pm S D$ & $9.5 \pm 1.8$ & $8.6 \pm 1.5$ & $10.3 \pm 1.7$ & $<0.001$ \\
\hline IVS (mm) M \pm SD & $10.7 \pm 2.4$ & $9.5 \pm 2.0$ & $11.6 \pm 2.2$ & $<0.001$ \\
\hline $\operatorname{EDD}(\mathrm{mm}) \mathrm{M} \pm \mathrm{SD}$ & $50.7 \pm 5.7$ & $48.4 \pm 5.2$ & $52.6 \pm 5.4$ & $<0.001$ \\
\hline $\mathrm{ESD}(\mathrm{mm}) \mathrm{M} \pm \mathrm{SD}$ & $31.2 \pm 5.5$ & $29.6 \pm 5.4$ & $32.6 \pm 5.3$ & 0.001 \\
\hline RWT (-) M \pm SD & $0.379 \pm 0.082$ & $0.359 \pm 0.079$ & $0.396 \pm 0.080$ & 0.003 \\
\hline $\operatorname{LVMI}\left(\mathrm{g} / \mathrm{m}^{2}\right) \mathrm{M} \pm \mathrm{SD}$ & $97.07 \pm 26.43$ & $83.20 \pm 19.84$ & $108.79 \pm 25.72$ & $<0.001$ \\
\hline $\operatorname{EDV}\left(\mathrm{cm}^{3}\right) \mathrm{M} \pm \mathrm{SD}$ & $119.0 \pm 29.1$ & $107.9 \pm 24.7$ & $128.3 \pm 29.5$ & $<0.001$ \\
\hline $\mathrm{ESV}\left(\mathrm{cm}^{3}\right) \mathrm{M} \pm \mathrm{SD}$ & $38.3 \pm 14.4$ & $33.1 \pm 12.9$ & $42.7 \pm 14.2$ & $<0.001$ \\
\hline $\mathrm{SV}\left(\mathrm{cm}^{3}\right) \mathrm{M} \pm \mathrm{SD}$ & $81.5 \pm 18.3$ & $74.7 \pm 15.9$ & $87.2 \pm 18.3$ & $<0.001$ \\
\hline $\mathrm{EF}(\%) \mathrm{M} \pm \mathrm{SD}$ & $68.6 \pm 5.9$ & $69.7 \pm 5.5$ & $68.0 \pm 6.1$ & 0.298 \\
\hline MFS (\%) M \pm SD & $17.7 \pm 2.9$ & $18.7 \pm 3.2$ & $17.0 \pm 2.5$ & $<0.001$ \\
\hline $\mathrm{S}^{\prime}(\mathrm{mm} / \mathrm{s}) \mathrm{M} \pm \mathrm{SD}$ & $8.1 \pm 1.6$ & $8.3 \pm 1.9$ & $8.0 \pm 1.5$ & 0.584 \\
\hline$e^{\prime}(\mathrm{cm} / \mathrm{s}) \mathrm{M} \pm \mathrm{SD}$ & $8.3 \pm 2.1$ & $8.7 \pm 2.2$ & $8.0 \pm 2.0$ & 0.038 \\
\hline$e^{\prime} / a^{\prime}(-) M \pm S D$ & $0.777 \pm 0.193$ & $0.808 \pm 0.195$ & $0.750 \pm 0.189$ & 0.014 \\
\hline$E / e^{\prime}(-) M \pm S D$ & $8.459 \pm 2.137$ & $7.907 \pm 1.627$ & $8.925 \pm 2.401$ & 0.015 \\
\hline
\end{tabular}

Table 2: Echocardiographic characteristics of the study groups.

No significant differences were found between women and men in terms of conventional LV systolic function indices such as $\mathrm{EF}$ and endocardial FS. Also, subendocardial longitudinal function expressed by $S^{\prime}$ did not differ between men and women with MS. Men had lower mean values of midwall FS $(\mathrm{P}<0.001)$ (Table 2$)$. LV DD was slightly more frequent in men than in women $(n=41,57.7 \%$ vs $n=29,48.3 \%)$ but this was not statistically significant $(\mathrm{P}=0.368)$. Diastolic function abnormalities were also most advanced in men compared with women-mean values of $e^{\prime}$ and $e^{\prime} / a^{\prime}$ were lower and $E / e^{\prime}$ ratio was higher in men than in women $(\mathrm{P}=0.038 ; \mathrm{P}=0.014$ and $\mathrm{P}=0.015$ respectively) (Table 2).

\section{Carotid arterial stiffness parameters in men and women with MS}

Mean values of AS parameters differed between men and women with MS. The mean values of $\beta$ and $\operatorname{PWV} \beta$ were higher in men comparing to women $(\mathrm{P}=0.02, \mathrm{P}=0.03$ respectively). Women had lower mean values of arterial compliance $(\mathrm{AC}, \mathrm{P}=0.018)$. Also, a measure of arterial total compliance (SV/PP) was significantly lower in women than in men $(\mathrm{P}=0.01)$ (Table 3$)$. 
Citation: Kruszyńska E, Kozakova M, Rudnicka ML, Morizzo C, Palombo C, et al. (2018) Predictors of Left Ventricular Diastolic Dysfunction in

\begin{tabular}{|c|c|c|c|c|}
\hline \multirow{2}{*}{ Arterial stiffness } & \multirow{2}{*}{ All $\mathrm{N}=131$} & \multicolumn{2}{|l|}{ Sex } & \multirow[t]{2}{*}{ W vs. M } \\
\hline & & Women $\mathrm{N}=\mathbf{6 0}$ & Men $\mathrm{N}=71$ & \\
\hline$\beta(-) M \pm S D$ & $9.06 \pm 3.43$ & $8.21 \pm 2.88$ & $9.79 \pm 3.71$ & 0.02 \\
\hline $\mathrm{Ep}(\mathrm{kPa}) \mathrm{M} \pm \mathrm{SD}$ & $128.1 \pm 55.5$ & $117.4 \pm 46.0$ & $137.1 \pm 61.3$ & 0.079 \\
\hline $\mathrm{AC}\left(\mathrm{mm}^{2} / \mathrm{kPa}\right) \mathrm{M} \pm \mathrm{SD}$ & $0.741 \pm 0.296$ & $0.697 \pm 0.315$ & $0.777 \pm 0.276$ & 0.018 \\
\hline$P W V \beta(m / s) M \pm S D$ & $6.38 \pm 0.96$ & $6.17 \pm 0.76$ & $6.56 \pm 1.08$ & 0.03 \\
\hline $\mathrm{SV} / \mathrm{PP}\left(\mathrm{cm}^{3} / \mathrm{mm} \mathrm{Hg}\right) \mathrm{M} \pm \mathrm{SD}$ & $1.466 \pm 0.422$ & $1.382 \pm 0.427$ & $1.537 \pm 0.407$ & 0.01 \\
\hline
\end{tabular}

Table 3: Carotid arterial stiffness indices in men and women with MS.

\section{Correlations of LV DD parameters in men and women with MS (chart)}

The following significant linear correlations of LV diastolic dysfunction indices were found in women: e' and age $(r=-0.407)$, e' and LVMI ( $r=-0.366)$, e' and AC ( $r=0.283)$, e' and PWV $\beta$ ( $r=-0.271) ; e^{\prime} / a^{\prime}$ and age $(r=-0.258)$, e'/a' and LVMI ( $r=-0.295) ; E / e^{\prime}$ and SBP $(r=0.259)$ $\mathrm{E} / \mathrm{e}^{\prime}$ and glucose $(\mathrm{r}=0.348), \mathrm{E} / \mathrm{e}^{\prime}$ and LVMI $(\mathrm{r}=0.296)$. In men linear regression analysis revealed the following significant linear correlations of LV diastolic dysfunction indices: e' and age $(r=-0.368)$, e' and LVMI $(\mathrm{r}=-0.277), \mathrm{e}^{\prime}$ and $\mathrm{AC}(\mathrm{r}=0.255) ; \mathrm{e}^{\prime} / \mathrm{a}^{\prime}$ and age $(\mathrm{r}=-0.301) ; \mathrm{E} / \mathrm{e}^{\prime}$ and glucose $(\mathrm{r}=0.311)$.

\section{ROC curve analysis of LV DD occurrence in men and women with MS}

With the use of ROC curve analysis, optimal cut-off values of parameters in the determination of diastolic dysfunction occurrence were identified (Table 4).

\begin{tabular}{|c|c|c|c|c|c|c|c|c|}
\hline & \multicolumn{4}{|l|}{ Women } & \multicolumn{4}{|l|}{ Men } \\
\hline & Cut-off & Sens. & Spec. & AUC & Cut-off & Sens. & Spec. & AUC \\
\hline & $>43$ & 85.5 & 48.5 & 0.711 & $>59$ & 46.3 & 90 & 0.719 \\
\hline $\mathrm{BMI}\left(\mathrm{kg} / \mathrm{m}^{2}\right)$ & $£ 32.5$ & 93.6 & 27.3 & 0.567 & $>29.0$ & 75.6 & 33.3 & 0.51 \\
\hline $\mathrm{HR}$ (bpm) & $£ 81$ & 90.8 & 14.6 & 0.493 & $>63$ & 75.6 & 40 & 0.54 \\
\hline $\mathrm{SBP}(\mathrm{mm} \mathrm{Hg})$ & $>122$ & 73.4 & 41.9 & 0.568 & $>135$ & 58.5 & 63.3 & 0.604 \\
\hline $\mathrm{DBP}(\mathrm{mm} \mathrm{Hg})$ & $£ 75$ & 53.8 & 54.5 & 0.515 & $<81$ & 36.6 & 83.3 & 0.546 \\
\hline $\mathrm{MBP}(\mathrm{mm} \mathrm{Hg})$ & $>99$ & 32.4 & 73.7 & 0.517 & $>102$ & 31.7 & 90 & 0.557 \\
\hline Glucose (mg/dL) & $>91$ & 86.7 & 44.9 & 0.618 & $>105$ & 82.9 & 36.7 & 0.579 \\
\hline Waist (cm) & $>99$ & 78.7 & 36.1 & 0.568 & $>112$ & 47.1 & 78.6 & 0.58 \\
\hline $\mathrm{HDL}(\mathrm{mg} / \mathrm{dL})$ & $<59$ & 61.2 & 68.6 & 0.632 & $<44$ & 55 & 80 & 0.612 \\
\hline TG (mg/dL) & $>104$ & 54.7 & 67.2 & 0.622 & $>214$ & 84.6 & 33.3 & 0.552 \\
\hline $\mathrm{PW}(\mathrm{mm}$ & $>8.9$ & 75.7 & 59.1 & 0.722 & $>10$ & 58.5 & 76.7 & 0.725 \\
\hline IVS (mm) & $>9.4$ & 75.1 & 69.2 & 0.777 & $>12.0$ & 58.5 & 83.3 & 0.748 \\
\hline RWT (-) & $>0.381$ & 65.9 & 82.3 & 0.774 & $>0.435$ & 43.9 & 86.7 & 0.68 \\
\hline $\operatorname{LVMI}\left(\mathrm{g} / \mathrm{m}^{2}\right)$ & $>80$ & 77.5 & 57.6 & 0.695 & $>115$ & 53.7 & 96.7 & 0.754 \\
\hline $\mathrm{PP}(\mathrm{mm} \mathrm{Hg})$ & $>55$ & 45.1 & 73.7 & 0.594 & $>58$ & 51.2 & 70 & 0.565 \\
\hline $\operatorname{SV} / P P(\mathrm{~mL} / \mathrm{mm} \mathrm{Hg})$ & $£ 1.71$ & 83.8 & 36.4 & 0.638 & $£ 1.79$ & 90.2 & 33.3 & 0.48 \\
\hline
\end{tabular}


Citation: Kruszyńska E, Kozakova M, Rudnicka ML, Morizzo C, Palombo C, et al. (2018) Predictors of Left Ventricular Diastolic Dysfunction in Metabolic Syndrome: Gender Differences. J Metabolic Synd 7: 244. doi:10.4172/2167-0943.1000244

Page 5 of 8

\begin{tabular}{|l|l|l|l|l|l|l|l|l|}
\hline$\beta(-)$ & $>6.3$ & 74 & 37.9 & 0.523 & $>8.8$ & 63.4 & 56.7 & 0.596 \\
\hline Ep $(\mathrm{kPa})$ & $>69$ & 84.4 & 24.2 & 0.529 & $>110$ & 68.3 & 56.7 & 0.611 \\
\hline AC $\left(\mathrm{mm}^{2} / \mathrm{kPa}\right)$ & $£ 0.87$ & 82.7 & 50 & 0.693 & $£ 0.64$ & 51.2 & 83.3 & 0.669 \\
\hline $\mathrm{PWV} \beta(\mathrm{m} / \mathrm{s})$ & $>6.4$ & 63.6 & 82.3 & 0.625 & $>6.6$ & 63.4 & 73.3 & 0.652 \\
\hline
\end{tabular}

Table 4: ROC curve analysis in the determination of LV DD occurrence in men and women with MS.

Univariable analysis proved the following significant variables in determining LV diastolic dysfunction in women (Table 5): age $>43$ yrs, $\mathrm{SBP}>122 \mathrm{mmHg}$, glucose level $>91 \mathrm{mg} \%$, HDL $<59 \mathrm{mg} \%$, TG $>104 \mathrm{mg}$ $\%$, LVMI $>80 \mathrm{~g} / \mathrm{m}^{2}, \quad \mathrm{RWT}>0,381, \quad \mathrm{MFS}<20 \%, \quad \mathrm{PP}>55, \quad \mathrm{SV} / \mathrm{PP}<1.71$, $\beta>6.3, \mathrm{PWV} \beta>6.5, \mathrm{AC}<0.87$. However, at multivariate analysis, only RWT was independently associated with LV DD occurrence in women with MS (Table 5).

\begin{tabular}{|c|c|c|c|c|c|c|c|}
\hline \multirow[b]{2}{*}{ Variables } & \multirow{2}{*}{$\begin{array}{l}\text { Cut- } \\
\text { off } \\
\text { value }\end{array}$} & \multicolumn{3}{|c|}{ Univariable analysis } & \multicolumn{3}{|c|}{ Multivariable analysis } \\
\hline & & OR & $\begin{array}{l}95 \% \\
\mathrm{Cl}\end{array}$ & $\begin{array}{l}\mathrm{P}- \\
\text { value }\end{array}$ & OR & $\begin{array}{l}95 \% \\
\mathrm{Cl}\end{array}$ & $\begin{array}{l}\mathrm{P}- \\
\text { value }\end{array}$ \\
\hline Age (years) & $>43$ & 5.57 & $\begin{array}{l}3.35-9 \\
.25\end{array}$ & $\begin{array}{l}<0.00 \\
1\end{array}$ & & & \\
\hline BMI $\left(\mathrm{kg} / \mathrm{m}^{2}\right)$ & $>31.5$ & 1.09 & $\begin{array}{l}0.90-1 \\
.33\end{array}$ & 0.354 & & & \\
\hline $\mathrm{HR}(\mathrm{bpm})$ & $£ 81$ & 1.68 & $\begin{array}{l}0.88-3 \\
.22\end{array}$ & 0.153 & & & \\
\hline 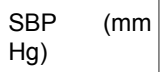 & $>122$ & 1.99 & $\begin{array}{l}1.28-3 \\
.09\end{array}$ & 0.003 & & & \\
\hline $\begin{array}{l}\text { DBP } \\
\mathrm{Hg})\end{array}$ & $£ 75$ & 1.39 & $\begin{array}{l}0.93-2 \\
.10\end{array}$ & 0.136 & & & \\
\hline $\begin{array}{l}\text { MBP } \quad(\mathrm{mm} \\
\mathrm{Hg})\end{array}$ & $>99$ & 1.34 & $\begin{array}{l}0.86-2 \\
.11\end{array}$ & 0.239 & & & \\
\hline $\begin{array}{l}\text { Glucose } \\
\text { (mg/dL) }\end{array}$ & $>91$ & 5.33 & $\begin{array}{l}3.16-8 \\
.96\end{array}$ & $\begin{array}{l}<0.00 \\
1\end{array}$ & & & \\
\hline Waist (cm) & $>99$ & 1.91 & $\begin{array}{l}1.18-3 \\
.09\end{array}$ & 0.011 & & & \\
\hline $\mathrm{HDL}(\mathrm{mg} / \mathrm{dL})$ & $<59$ & 3.44 & $\begin{array}{l}2.20-5 \\
.36\end{array}$ & $\begin{array}{l}<0.00 \\
1\end{array}$ & & & \\
\hline TG $(\mathrm{mg} / \mathrm{dL})$ & $>104$ & 2,48 & $\begin{array}{l}1,52-4 \\
, 05\end{array}$ & $\begin{array}{l}<0,00 \\
1\end{array}$ & & & \\
\hline $\mathrm{PW}(\mathrm{mm})$ & $>8.9$ & 4.51 & $\begin{array}{l}2.88-7 \\
.05\end{array}$ & $\begin{array}{l}<0.00 \\
1\end{array}$ & & & \\
\hline IVS (mm) & $>9.4$ & 6.63 & $\begin{array}{l}4.20-1 \\
0.5\end{array}$ & $\begin{array}{l}<0.00 \\
1\end{array}$ & & & \\
\hline RWT (-) & $\begin{array}{l}>0.38 \\
1\end{array}$ & 9 & $\begin{array}{l}5.56-1 \\
4.6\end{array}$ & $\begin{array}{l}<0.00 \\
1\end{array}$ & 5,87 & $\begin{array}{l}1.46-2 \\
3.5\end{array}$ & 0.012 \\
\hline LVMI $\left(\mathrm{g} / \mathrm{m}^{2}\right)$ & $>80$ & 4.66 & $\begin{array}{l}2.96-7 \\
.35\end{array}$ & $\begin{array}{l}<0.00 \\
1\end{array}$ & & & \\
\hline $\mathrm{PP}(\mathrm{mm} \mathrm{Hg})$ & $>55$ & 2.31 & $\begin{array}{l}1.49-3 \\
.56\end{array}$ & $\begin{array}{l}<0.00 \\
1\end{array}$ & & & \\
\hline $\begin{array}{l}\text { SV/PP } \\
(\mathrm{mL} / \mathrm{mm} \mathrm{Hg})\end{array}$ & $£ 1.71$ & 2.72 & $\begin{array}{l}1.66-4 \\
.46\end{array}$ & $\begin{array}{l}<0.00 \\
1\end{array}$ & & & \\
\hline
\end{tabular}

\begin{tabular}{|l|l|l|l|l|l|l|l|}
\hline EF (\%) & $>68.0$ & 0.8 & $\begin{array}{l}0.15-4 \\
.26\end{array}$ & 0.873 & & & \\
\hline MFS\% & $£ 20.0$ & 4.5 & $\begin{array}{l}1.36-1 \\
4.8\end{array}$ & 0,022 & & & \\
\hline$S^{\prime}(\mathrm{mm} / \mathrm{s})$ & $<8.6$ & 12 & $\begin{array}{l}0.98-1 \\
37\end{array}$ & 0.084 & & & \\
\hline$\beta(-)$ & $>6.3$ & 1.73 & $\begin{array}{l}1.11-2 \\
.71\end{array}$ & 0.02 & & & \\
\hline Ep (kPa) & $>69$ & 1.73 & $\begin{array}{l}0.99-2 \\
.92\end{array}$ & 0.053 & & & \\
\hline $\begin{array}{l}\text { AC }(\mathrm{mm} 2 / \\
\mathrm{kPa})\end{array}$ & $£ 0.87$ & 4.77 & $\begin{array}{l}2.94-7 \\
.72\end{array}$ & $<0.00$ & & & \\
\hline $\mathrm{PWV} \beta(\mathrm{m} / \mathrm{s})$ & $>6.5$ & 8.13 & $\begin{array}{l}5.04-1 \\
3.1\end{array}$ & $<0.00$ & & & \\
\hline
\end{tabular}

Table 5: Multiple logistic regression analysis for the discrimination of left ventricular diastolic dysfunction (women with MS).

In univariable analysis provided in men with MS, it turned out that significant determinants of LV diastolic dysfunction in men were (Table 6): age $>59$ yrs, $\mathrm{HDL}<44 \mathrm{mg} \%$, LVMI $>115 \mathrm{~g} / \mathrm{m}^{2}$, RWT $>0,435$, $\mathrm{S}^{\prime}<7.7 \mathrm{~cm} / \mathrm{s} ; \quad \mathrm{SV} / \mathrm{PP}<1.79, \quad \mathrm{PWV} \beta>6.6, \mathrm{AC}<0.64$. At multivariate analysis, only RWT and LVMI, as well as AC, were independently associated with LV DD occurrence in men.

\begin{tabular}{|c|c|c|c|c|c|c|c|}
\hline \multirow[b]{2}{*}{ Variables } & \multirow[t]{2}{*}{$\begin{array}{l}\text { Cut- } \\
\text { off } \\
\text { value }\end{array}$} & \multicolumn{3}{|c|}{ Univariable analysis } & \multicolumn{3}{|c|}{ Multivariable analysis } \\
\hline & & OR & $\begin{array}{l}95 \% \\
\mathrm{Cl}\end{array}$ & $\begin{array}{l}\mathrm{P} \text { - } \\
\text { value }\end{array}$ & OR & $\begin{array}{l}95 \% \\
\mathrm{Cl}\end{array}$ & $\begin{array}{l}P- \\
\text { value }\end{array}$ \\
\hline Age (years) & $>59$ & 7.77 & $\begin{array}{l}2.03- \\
29.7\end{array}$ & 0.003 & & & \\
\hline BMI $\left(\mathrm{kg} / \mathrm{m}^{2}\right)$ & $>29.0$ & 1.33 & $\begin{array}{l}0.46- \\
3.82\end{array}$ & 0.798 & & & \\
\hline $\mathrm{HR}(\mathrm{bpm})$ & $>63$ & 2.07 & $\begin{array}{l}0.74- \\
5.73\end{array}$ & 0.252 & & & \\
\hline $\begin{array}{l}\text { SBP } \quad(\mathrm{mm} \\
\mathrm{Hg})\end{array}$ & $>135$ & 1.41 & $\begin{array}{l}0.55- \\
3.64\end{array}$ & 0.636 & & & \\
\hline $\begin{array}{l}\text { DBP } \quad(\mathrm{mm} \\
\mathrm{Hg})\end{array}$ & $>81$ & 2.88 & $\begin{array}{l}0.91- \\
9.12\end{array}$ & 0.115 & & & \\
\hline $\begin{array}{l}\text { MBP } \quad(\mathrm{mm} \\
\mathrm{Hg})\end{array}$ & $>102$ & 4.18 & $\begin{array}{l}0.99- \\
16.3\end{array}$ & 0.061 & & & \\
\hline $\begin{array}{l}\text { Glucose } \\
\text { (mg/dL) }\end{array}$ & $>105$ & 2.81 & $\begin{array}{l}0.93- \\
8.46\end{array}$ & 0.11 & & & \\
\hline
\end{tabular}




\begin{tabular}{|c|c|c|c|c|c|c|c|}
\hline Waist $(\mathrm{cm})$ & $>112$ & 4.09 & $\begin{array}{l}1.26- \\
13.3\end{array}$ & 0.032 & & & \\
\hline $\begin{array}{l}\mathrm{HDL} \\
(\mathrm{mg} / \mathrm{dL})\end{array}$ & $<44$ & 4.89 & $\begin{array}{l}1.64- \\
14.5\end{array}$ & 0.007 & & & \\
\hline $\mathrm{TG}(\mathrm{mg} / \mathrm{dL})$ & $>214$ & 2,75 & $\begin{array}{l}0,87- \\
8,72\end{array}$ & 0,143 & & & \\
\hline $\mathrm{PW}(\mathrm{mm})$ & $>10$ & 4.64 & $\begin{array}{l}1.62- \\
13.3\end{array}$ & 0.007 & & & \\
\hline IVS (mm) & $>12$ & 7.06 & $\begin{array}{l}2.25- \\
22.2\end{array}$ & 0.001 & & & \\
\hline RWT (-) & $\begin{array}{l}>0.43 \\
5\end{array}$ & 5.09 & $\begin{array}{l}1.50- \\
17.2\end{array}$ & 0.013 & 8.34 & $\begin{array}{l}1.73- \\
40.1\end{array}$ & 0.008 \\
\hline LVMI $\left(\mathrm{g} / \mathrm{m}^{2}\right)$ & $>115$ & 33.6 & $\begin{array}{l}4.17- \\
270\end{array}$ & $\begin{array}{l}<0.00 \\
1\end{array}$ & 38.8 & $\begin{array}{l}4.14- \\
364\end{array}$ & 0.001 \\
\hline $\mathrm{PP}(\mathrm{mm} \mathrm{Hg})$ & $>58$ & 2.45 & $\begin{array}{l}0.91- \\
6.61\end{array}$ & 0.122 & & & \\
\hline $\begin{array}{l}\text { SV/PP } \\
(\mathrm{mL} / \mathrm{mm} \mathrm{Hg})\end{array}$ & $£ 1.79$ & 4.62 & $\begin{array}{l}1.28- \\
16.6\end{array}$ & 0.03 & & & \\
\hline $\mathrm{EF}(\%)$ & $>59.8$ & 3.38 & $\begin{array}{l}0.67- \\
16.9\end{array}$ & 0.267 & & & \\
\hline MFS $\%$ & $£ 17.3$ & 2.73 & $\begin{array}{l}0.99- \\
7.37\end{array}$ & 0.08 & & & \\
\hline $\mathrm{S}^{\prime}(\mathrm{mm} / \mathrm{s})$ & $£ 7.7$ & 8.71 & $\begin{array}{l}1.64- \\
46.3\end{array}$ & 0.014 & & & \\
\hline$\beta(-)$ & $>8.8$ & 2.27 & $\begin{array}{l}0.87- \\
5.93\end{array}$ & 0.15 & & & \\
\hline $\mathrm{Ep}(\mathrm{kPa})$ & $>110$ & 2.82 & $\begin{array}{l}0.96- \\
7.48\end{array}$ & 0.063 & & & \\
\hline $\begin{array}{l}\mathrm{AC} \\
\mathrm{kPa})\end{array}$ & $£ 0.64$ & 5.25 & $\begin{array}{l}1.68- \\
16.4\end{array}$ & 0.006 & 9.04 & $\begin{array}{l}2.07- \\
39.6\end{array}$ & 0.004 \\
\hline$P W V \beta(m / s)$ & $>6.6$ & 4.77 & $\begin{array}{l}1.70- \\
13.3\end{array}$ & 0.005 & & & \\
\hline
\end{tabular}

Table 6: Multiple logistic regression analysis for the discrimination of left ventricular diastolic dysfunction (men with MS).

\section{Discussion}

Metabolic syndrome contributes to the development of cardiovascular events [19]. Cardiovascular involvement associated with MS include vascular and myocardial abnormalities that are initially manifested as increased large artery stiffness and impaired relaxation of the left ventricle and has a prognostic importance in pts with MS [20]. The correlation between the intensity of MS and both the presence and grade of DD is reported in several studies [21,22]. The potent well-known determinants of LV DD in various conditions are aging, hypertension, diabetes, obesity, LVH and LV systolic function [10]. Recently also AS has been being considered as a novel predictor of LV DD [6-10]. To the best of our knowledge, this is the first study to determine sex-specific predictors of LV DD in MS.

\section{Aging as a predictor of LV DD in men and women with MS}

Age is an established predictor of LV DD both in men and women in many conditions [10]. Some authors have shown that aging may have a greater impact on LV DD occurrence in women than in men [23]. In contrast, in our previous study of untreated hypertensives, age was documented as a significant determinant of LV DD only in men [10]. In the present study, age was identified as a predictor of LV DD in both women and men with MS in the univariate analysis provided in separate sex subgroups (Tables 5 and 6). What is interesting, the cut-off point of age as a predictor of LV DD was much lower for women than men (43 yrs vs. 59 yrs) suggesting that even younger women with MS may be prone to LV DD development.

\section{Left ventricular hypertrophy as a predictor of LVDD in men and women with MS}

One of the powerful sex-specific cardiovascular factors is LVMI, which is usually greater in men. In our study, male patients presented with higher values of LVMI $(\mathrm{P}<0.001)$ (Table 1$)$. LVH is an established predictor of LV DD in various populations [16]. In the present study, univariate analysis in separate sex subgroups (Tables 5 and 6) revealed that LVH indices, namely, RWT and LVMI were significant predictors of LV DD both in men and women with MS. Multivariate analysis provided in separate sex subgroups proved LVH in a feature of LVMI and RWT as an independent predictors of LV DD in men with MS and RWT only as an independent determinant of LV DD in women with MS. Our previous study performed in pts with untreated hypertension showed LVH indices as predictors of LV DD in men and not in women [10]. In contrast, Nicolini et al. showed that the effects of MS on LV concentric hypertrophy/remodeling and preclinical diastolic dysfunctions were more pronounced in women [3].

\section{LV systolic function and LV DD in men and women}

LV DD has been shown to be associated with long-axis systolic dysfunction in various populations [24]. The present study showed in the univariate analysis $S^{\prime}$ as a predictor of LVDD in men but not in women (Tables 4 and 5). In addition, in our previous study of untreated hypertensives $S^{\prime}$ was proved in the multivariate analysis performed in the separate sex subgroups as an independent predictor of LV DD in men, but not in women [10]. That was the first study in which LV long-axis systolic dysfunction was identified as a sex-specific predictor of LV DD in untreated hypertension as this is the first study exploring the role of LV subendocardial longitudinal systolic function as a sex-specific predictor of LV DD in MS. Why $\mathrm{S}^{\prime}$ influenced the occurrence of LV DD in men to a larger extent than in women. One potential reason is a higher prevalence of silent ischemia in men.

LV Midwall Fractional Shortening (MFS) is more precised than LV endocardial fractional shortening in diagnosing an early LV systolic dysfunction in patients with LVH and hypertension [15]. Women with MS have a depressed afterload-corrected midwall fractional shortening than women without the metabolic syndrome, whereas no differences emerged in men with MS in the study by Schillaci et al. [25]. The present study showed in the univariate analysis MFS as a predictor of LVDD in women but not in men however the mean values of $\mathrm{m} \mathrm{FS}$ were lower in men comparing to women with MS. We have not met before the study examining the role of MFS as a predictor of LV DD in MS.

\section{Arterial stiffness as a predictor of LV DD in men and women}

Women have a stronger association of baPWV with metabolic syndrome than men [12]. Conversely, data from MARK study showed a greater association of CAVI and baPWV with MetS components in 
men than in women and indicated greater arterial stiffness in the event of elevated blood pressure, fasting plasma glucose and waist circumference [26]. Consistently, in our study, the mean values of AS parameters namely $\beta$ and $\mathrm{PWV} \beta$ were higher in men compared to women $(\mathrm{P}=0.02, \mathrm{P}=0.03$ respectively) and women have lower mean values of $\mathrm{AC}(\mathrm{P}=0.018)$ and a measure of arterial total compliance$\mathrm{SV} / \mathrm{PP})(\mathrm{P}=0.01)$ (Table 3$)$. LV DD is not only a disease of a stiff ventricle but also a disorder of stiff arteries. It is also considered as a coupling disease with disturbed arterial-ventricular interactions.

The relationship between AS and LV DD has been previously reported in pts with hypertension [10], in diabetes [20] and in elderly [27]. It has been also proved by Kass et al. [7] that increased AS accounts for diminished exercise tolerance in HFpEF pts. Growing evidence suggests that an increase in AS and related arterialventricular coupling may play an important role in increasing cardiovascular risk in MS [28]. Recently Solovjova et al. proved AS measured by carotid-femoral PWV as an independent determinant of LV DD in MS pts [29]. To the best of our knowledge, this is the first study to examine sex-differences in AS as a predictor of LV DD in MS. The univariate analysis of this study revealed that increased AS and decreased AC was associated with LV DD occurrence in both women and men. AC was proved as an independent determinant of LV DD occurrence only in men. Also, our previous study performed in untreated hypertensive proved AS as an independent determinant of LV DD in men but not in women [30].

\section{Conclusion}

There are sex-differences in LV DD predictors in MS. Besides LVH as a powerful determinant of LVDD in both men and women with MS, also arterial compliance as a measure of arterial stiffness was independently associated with LV DD occurrence in men but not in women with MS.

\section{Clinical Implications}

MS is not considered, so far, a gender-specific cause of cardiovascular risk. Nevertheless, there is new evidence that significant gender differences are present in the relationship between MS and cardiovascular diseases. Detecting gender-related differences of cardiac remodeling, including LV DD in MS would be of major importance to improve the prevention, diagnosis, and treatment of these patients.

\section{Declarations}

\section{Ethics approval and consent to participate}

The study was approved by the Ethics Committee of Wroclaw University of Medicine and University of Pisa; written informed consent was sought from each participant.

\section{Authors contribution}

EK, JJ and KL-G conceived the idea for the study. EK, JJ, and KL-G contributed to the study design. All authors were involved in data collection. EK, JJ, CP, and KL-G analyzed the data. MK and CP provided a critical review. All authors edited and approved the final version of the manuscript.

\section{References}

1. Motillo S, Filion KB, Genest J, Joseph L, Pilote 1, et al. (2010) The metabolic syndrome and cardiovascular risk: a systematic review and meta-analysis. J Am Coll Cardiol 56: 1113-11132.

2. Schillaci G, Verdecchia P, Porcellati C, Cuccurullo O, Cosco C, et al. (2000) Continuous relations between left ventricular mass and cardiovascular risk in essential hypertension. Hypertension 35: 580-586.

3. Nicolini E, Martegani G, Maresca AM, Marchesi C, Dentali F, et al. (2013) Left ventricular remodeling in patients with metabolic syndrome: influence of gender. Nutrition, Metabolism and Cardiovascular Diseases 23: 771-775.

4. Schillaci G, Pasqualini L, Verdecchia P, Vaudo G, Marchesi S, et al. (2002) Prognostic significance of left ventricular diastolic dysfunction in essential hypertension. J Am Coll Cardiol 39: 2005-2011.

5. Hunt KJ, Resendez RG, Williams K, Haffner SM, Stern MP (2004) San Antonio Heart Study. National cholesterol education program versus world health organization metabolic syndrome in relations to all-cause and cardiovascular mortality in San Antonio. heart study. Circulation 110: 1251-1257.

6. Sarafidis PA, Mc Farlane SI, Bakris GL (2006) Gender disparity in outcomes of care and management for diabetes and metabolic syndrome. Curr Diabetol Rep 6: 219-224.

7. Kawaguchi M, Hay I, Fetics B, Kass DA (2003) Combined ventricular systolic and arterial stiffening in patients with heart failure and preserved ejection fraction: implications for systolic and diastolic reserve limitations. Circulation 107: 714-720.

8. Borlaug BA, Paulus WJ (2011) Heart failure with preserved ejection fraction: pathophysiology, diagnosis and treatment. Eur Heart J 32: 670-679.

9. Vriz O, Bossone E, Bettio M, Pavan D, Carerj S (2011) Carotid artery stiffness and diastolic function in subjects without known cardiovascular disease. J Am Soc Echocardiogr 24: 915-921.

10. Jaroch J, Loboz Grudzien K, Bociaga Z (2012) The relationship of carotid arterial stiffness to left ventricular diastolic dysfunction in untreated hypertension. Kardiol Pol 70: 223-231.

11. Jaroch J, Vriz O, Bociąga Z, Driussi C, Loboz-Rudnicka M (2016) Sexspecific predictors of left ventricular diastolic dysfunction in untreated hypertension. Clin Interv Aging 11: 1495-1504.

12. Weng C, Yuan H, Yang K (2013) Gender-specific association between the metabolic syndrome and arterial stiffness in 8,300 subjects. Am J Med Sci 346: 289-294.

13. Kim HL, Lee JM, Seo JB (2015) The effects of metabolic syndrome and its components on arterial stiffness in relation to gender. J Cardiol 65: 243-249.

14. Scuteri A, Cunha PG, Rosei EA, Badariere J, Bekaert S, et al. (2014) Arterial stiffness and influences of metabolic syndrome: a cross-country study. Atherosclerosis 233: 654-660.

15. Devereux RB, Alonso DR, Lutas EM, Gottlieb GJ, Campo E, et al. (1986) Echocardiographic assessment of left ventricular hypertrophy: comparison to necropsy findings. Am J Cardiol 57: 450-458.

16. De Simone G, Devereux RB, Roman MJ (1994) Assessment of left ventricular function by the midwall farctional shortening/end-systolic stress relation in human hypertension. J Am Coll Cardiol 23: 1444-1451.

17. Nagueh SF, Appleton ChP, Gillebert TC (2009) Recommendations for the evaluation of left ventricular diastolic function by echocardiography. Eur J Echocardiogr 10: 165-193.

18. Sugawara M, Niki K, Furuhata H, Ohnishi S, Suzuki S, et al. (2000) Relationship between the pressure and diameter of the carotid artery in humans. Heart Vessels 15: 49-51.

19. Magda SL, Ciobanu AO, Florescu M, Vinereanu D (2013) Comparative reproducibility of the noninvasive ultrasound methods for the assessment of vascular function. Heart Vessels 28: 143-150.

20. Miranda PJ, DeFronzo RA, Califf RM, Guyton JR (2005) Metabolic syndrome: evaluation of pathological and therapeutic outcomes. Am Heart J 149: 20-32. 
Citation: Kruszynska E, Kozakova M, Rudnicka ML, Morizzo C, Palombo C, et al. (2018) Predictors of Left Ventricular Diastolic Dysfunction in

21. Von Bibra H, St John Sutton M (2010) Diastolic dysfunction in diabetes and metabolic syndrome: Promising potential for diagnosis and prognosis. Diabetologia 53: 1033-1045.

22. De las Fuentes L, Brown AL, Mathews SJ, Waggoner AD (2007) Metabolic syndrome is associated with abnormal left ventricular diastolic dysfunction independent of left ventricular mass. Eur Heart J 28: 553-559.

23. Barylski M, Ciecwierz J, Banach M, Irzmanski R, Pawlicki L (2008) Left ventricular diastolic dysfunction in subjects with metabolic syndrome. Atherosclerosis 9: 232 .

24. Gori M, Lam CSP, Gupta DK (2014) Sex-specific cardiovascular structure and function in heart failure with preserved ejection fraction. Eur J Heart Fail 16: S35-S42.

25. Schillaci G, Pirro M, Pucci G (2006) Different impact of the metabolic syndrome on left ventricular structure and function in hypertensive men and women. Hypertension 47: 881-886.

26. Vinereanu D, Nicolaides E, Tweddel AC, Fraser AG (2000) "Pure" diastolic dysfunction is associated with long-axis systolic dysfunction: implications for the diagnosis and classification of heart failure. Eur J Heart Fail 7: 820-828.

27. Gomez Sanchez L, Garcia-Ortiz L, Patino Alonso MC (2016) Association of metabolic syndrome and its components with arterial stiffness in Caucasian subjects of the MARK study: a cross-sectional trial. Cardiovasc Diabetol 15: 148

28. Abhayaratna WP, Barnes ME, O'Rourke MF, Gersh BJ, Seward JB, et al (2006) Relation of arterial stiffness to left ventricular diastolic function and cardiovascular risk prediction in patients $\geq 65$ years of age. Am J Cardiol 98: 1387-1392.

29. Solovjova S, Ryliskyte L, Celutkiene J, Badariene J, Navickas R, et al. (2016) Aortic stiffness is an independent determinant of left ventricular diastolic dysfunction in metabolic syndrome patients. Blood Press 25: 11-20.

30. Chantler PD, Lakatta EG (2012) Arterial-ventricular coupling with aging and disease. Front Physiol 3: 90. 